- Original Paper •

\title{
Characterizing the Relative Importance Assigned to Physical Variables by Climate Scientists when Assessing Atmospheric Climate Model Fidelity
}

\author{
Susannah M. BURROWS*, Aritra DASGUPTA, Sarah REEHL, Lisa BRAMER, \\ Po-Lun MA, Philip J. RASCH, and Yun QIAN
}

Pacific Northwest National Laboratory, Richland, Washington 99354, USA

(Received 8 December 2017; revised 13 March 2018; accepted 3 April 2018)

\begin{abstract}
Evaluating a climate model's fidelity (ability to simulate observed climate) is a critical step in establishing confidence in the model's suitability for future climate projections, and in tuning climate model parameters. Model developers use their judgement in determining which trade-offs between different aspects of model fidelity are acceptable. However, little is known about the degree of consensus in these evaluations, and whether experts use the same criteria when different scientific objectives are defined. Here, we report on results from a broad community survey studying expert assessments of the relative importance of different output variables when evaluating a global atmospheric model's mean climate. We find that experts adjust their ratings of variable importance in response to the scientific objective, for instance, scientists rate surface wind stress as significantly more important for Southern Ocean climate than for the water cycle in the Asian watershed. There is greater consensus on the importance of certain variables (e.g., shortwave cloud forcing) than others (e.g., aerosol optical depth). We find few differences in expert consensus between respondents with greater or less climate modeling experience, and no statistically significant differences between the responses of climate model developers and users. The concise variable lists and community ratings reported here provide baseline descriptive data on current expert understanding of certain aspects of model evaluation, and can serve as a starting point for further investigation, as well as developing more sophisticated evaluation and scoring criteria with respect to specific scientific objectives.
\end{abstract}

Key words: climate, climate model, model evaluation, numerical model skill, expert elicitation

Citation: Burrows, S. M., A. Dasgupta, S. Reehl, L. Bramer, P.-L. Ma, P. J. Rasch, and Y. Qian, 2018: Characterizing the relative importance assigned to physical variables by climate scientists when assessing atmospheric climate model fidelity. Adv. Atmos. Sci., 35(9), 1101-1113, https://doi.org/10.1007/s00376-018-7300-x.

\section{Introduction}

A critical aspect of any climate modeling research is an evaluation of the realism, or fidelity, of the model's simulated climate through a careful comparison with observational data. For the purposes of this discussion, we define a climate model's "fidelity" broadly as the agreement of the simulated climate with the observed historical and presentday climate state, typically using a combination of satellite and ground-based observations, field campaign measurements, and reanalysis data products as primary sources of observational data. At climate modeling centers around the world, the development of a new model version is always followed by a calibration ("tuning") effort aimed at selecting values for model parameters that are physically justifiable and lead to a credible simulation of climate (Hourdin et al., 2017). Model tuning involves the completion of a large

\footnotetext{
${ }^{*}$ Corresponding author: Susannah M. BURROWS

Email: susannah.burrows@pnnl.gov
}

number of simulations with variations in parameters, input files, and other features of the model. Each simulation is painstakingly evaluated, typically by examining a set of priority metrics, accompanied by manual inspection of a variety of plots and visualizations of various modeled fields, and detailed comparisons to determine which model configuration produces a credible realization of the climate. Tuning one coupled climate model requires thousands of hours of effort by skilled experts. Experts must exercise judgment, based on years of training, experience, and broad and deep understanding of the model, the physical climate system, and observational constraints, in determining which trade-offs are defensible when different optimization goals conflict.

Comparisons of model fidelity across multiple model simulations are also carried out in multi-model intercomparison projects (e.g., Gleckler et al., 2008; Reichler and Kim, 2008), and in perturbed parameter ensemble experiments for the purpose of quantifying model uncertainty or sensitivities (Yang et al., 2013; Qian et al., 2015, 2016). Such studies aim to understand what factors lead to inter-model diversity and 
drive model sensitivities and to identify potential improvements. Additionally, if an adequate single metric of overall climate model fidelity could be developed, it could be applied to construct weighted averages of climate simulation ensembles (Min and Hense, 2006; Suckling and Smith, 2013), and used in automatic parameter optimization algorithms (Zhang et al., 2015).

Early efforts to characterize multi-variable climate model fidelity calculated an index of climate model fidelity by calculating a normalized root-mean-square error or similar metric for each of a selected set of model variables, and then averaging these metrics for all variables (Gleckler et al., 2008; Reichler and Kim, 2008). More nuanced objective methods have been proposed to account for the inherent variability in each field (Braverman et al., 2011), and for spatial and temporal dependencies between variables (Nosedal-Sanchez et al., 2016).

These objective methods characterize how closely models resemble observations of specific variables with an increasing degree of sophistication. Nevertheless, in all such approaches, expert judgement is exercised in the selection of which variables to include. In addition, in most previous studies, an implicit decision was made to treat all variables as being of equal physical importance. By contrast, when experts evaluate model fidelity, their decision-making implicitly incorporates their understanding of the physical importance of specific variables to the science questions they are interested in, and more emphasis is placed on the most physically relevant variables. Recent studies have emphasized that the selection of assessed variables should reflect physical understanding of the system under consideration (Knutti et al., 2017) and that different research teams may select different optimization criteria when weighting model ensemble members, depending on their goals (Herger et al., 2017).

A potential path forward is to construct a fidelity index $I$ that combines multiple metrics $m_{i}$ that characterize different aspects of model fidelity, weighted by their relative importance $w_{i}$ :

$$
I=\Sigma_{i} w_{i} m_{i}
$$

However, since the relative "importance" of different optimization goals is inherently subjective, any such index, including one in which all $w_{i}$ are equal, will be susceptible to criticism that the weights chosen are arbitrary.

Since expert judgement cannot be fully eliminated from the model evaluation process, we propose that it would be valuable to better understand and quantify the relative importance climate modelers assign to different aspects of model fidelity when making decisions about trade-offs. In addition, we believe it is important to quantify the degree to which consensus exists about the importance of such variables. In the longer term, we envision that this information can be used to develop metrics that quantify both the mean and the variability of the community's judgements about climate model fidelity.

This paper reports on our first step towards this long-term goal: the establishment of a baseline understanding of the level of importance that experts explicitly state they assign to different variables when evaluating the mean climate state of the atmosphere of a climate model. To this end, we conducted a large international survey of climate model developers and users, and asked them to indicate their view of the relative importance of a subset of variables used in assessing model fidelity, in the context of particular scientific goals. The specific aims of this study are to: (1) quantify the extent of consensus among climate modelers on the relative importance of different variables in evaluating climate models; (2) document whether modelers adjust their importance weights depending on the scientific purpose for which a model is being evaluated; (3) determine whether either importance rankings or degree of consensus vary as a function of an individual's experience or domain of expertise; and (4) provide baseline information for a planned follow-up study, a mock model evaluation exercise. In the follow-up study, described in more detail in section 4 , we will investigate whether experts' assessments of models, on the basis of plots and metrics describing model-observation comparisons, are consistent with the relative importance that these experts previously assigned to individual variables for the assessment of model fidelity, with respect to specific science goals.

We describe the present study in the following sections. Section 2 describes the design of the survey, recruitment of participants, and methods used in analyzing survey responses. Section 3 describes the results of the survey, including the distribution of importance rankings, degree of consensus, dependence of responses on the specific science questions and respondents' level of experience, and perceived barriers to systematic quantification of climate model fidelity. Section 4 discusses a potential approach to synthesizing expert assessments of model fidelity and objective methods for fidelity assessment, by systematically measuring and explicitly accounting for the relative importance experts assign to different aspects of fidelity. Finally, section 5 summarizes the key points and conclusions from this study.

\section{Survey design and methods}

\subsection{Survey aims, design and scope}

We conducted a large international survey to document and understand the expert judgments of the climate modeling community on the relative importance of different model variables in the evaluation of simulation fidelity.

To keep the scope of this study focused, we only considered the evaluation of the annual mean climatology of an atmosphere-only model simulation, with prescribed SST. In addition, participants were asked to assume that their evaluation would be carried out only on the basis of scalar metrics (e.g., RMSE, correlation) characterizing the agreement of the respective model field with observations.

Transient features of climate were intentionally excluded from this study, but are of critical importance in model evaluation, and should be explored in future work. Similarly, coupled climate models have more complex tuning criteria that 
are not considered here.

We chose to limit the number of variables and criteria under consideration in order to encourage broader participation, and in anticipation of a planned follow-up study (described in more detail in section 4). Briefly, the follow-up study will invite experts to compare and evaluate climate model outputs, and will aim to infer the importance that experts implicitly assign to different aspects of model fidelity in conducting this assessment. To the best of our knowledge, this would be the first attempt to experimentally characterize expert evaluations of climate model fidelity, and so we aim to initially test the approach using a small number of key variables, which will allow for a more controlled study. The relative importance ratings and other input from experts reported in this study will both inform the design of the follow-up study and provide $a$ priori values for Bayesian inference of the weights $w_{i}$.

The importance of a particular variable in model evaluation will depend on the purpose for which the model will be used. To better constrain the responses, as well as to explore how expert rankings of different model variables might change depending on the scientific objectives, we asked participants to rate the importance of different variables with respect to several different "Science Drivers". A list of the six Science Drivers used in this survey is shown in Table 1. For each Science Driver, participants were presented with a preselected list of variables thought to be relevant to that topic, and asked to rate the importance of each variable on a sevenpoint Likert scale from "Not at all Important" to "Extremely Important". Participants were also invited to provide written feedback identifying any "very important" or "extremely important" variables that they felt had been overlooked; many took the opportunity to provide these comments, summarized in Tables S1-S3 (see Electronic Supplementary Material). This feedback will be used to improve the survey design in the follow-up study.

\subsection{Survey recruitment, participation, and data screening}

The survey was distributed via several professional mailing lists targeting communities of climate scientists, especially model developers and users, and by directly soliciting input from colleagues through the professional networks of the authors of this paper. Due to privacy restrictions, we are unable to report the identities or geographic locations of

Table 1. Science Driver (SD) questions posed in this survey.

SD 1 How well does the model reproduce the overall features of the Earth's climate?

SD 2 How well does the model reproduce features of the global water cycle?

SD 3 How well does the model simulate Southern Ocean climate?

SD 4 How well does the model simulate important features of the water cycle in the Amazon watershed?

SD 5 How well does the model simulate important features of the water cycle in the Asian watershed?

SD 6 How well does the model simulate the climate impact of clouds globally? survey respondents, but we are confident that they are representative of the climate modeling community. The survey was open from 18 January 2017 to 25 April 2017. Participants who had not completed at least all items on the first Science Driver $(N=12)$, and participants who rated themselves as "not at all experienced" with evaluating model fidelity $(N=7)$ were excluded from analysis. Of the remaining 96 participants, 81 had completed all six Science Drivers.

Our survey respondents were a highly experienced group, with the vast majority of participants rating themselves as either "very familiar" (40.6\%) or "extremely familiar" (40.6\%) with climate modeling. In addition, a large fraction of our participants had worked in climate modeling for many years, with the majority of participants (62) reporting at least 10 years' experience, and a substantial number of participants (31) reporting at least 20 years' experience with climate modeling. When asked to rate their experience in "evaluating the fidelity of the atmospheric component of global climate model simulations," $37.5 \%$ rated themselves as "very experienced," and $20.8 \%$ as "moderately experienced" in "tuning/calibrating the atmospheric component of global climate model simulations". An overview of the characteristics of the survey participants is shown in Fig. 1.

\subsection{Formal consensus measure: Coefficient of Agree- ment (A)}

To quantify the degree of consensus among our participants, we employ a formal measure of consensus called the coefficient of agreement $A$ (Riffenburgh and Johnstone, 2009), which varies from values near 0 (no agreement; random responses) to a maximum possible value of 1 (complete consensus). Calculated values of $A$ for the two experience groups, and their probability $p$ of being significantly different from each other, are tabulated for all Science Drivers and variables in the Supplementary Tables S4-S6.

The coefficient of agreement is calculated from the observed disagreement $d_{\mathrm{obs}}$ and the expected disagreement under the null hypothesis of random responses $d_{\text {exp }}$. Let $r_{\max }$ denote the number of possible options ( 7 in the Likert scale used here); let $r=1 \ldots r_{\max }$ denote the possible responses ( $r=7$ is "Extremely important", $r=6$ is "Very important", and so on); let $n_{r}$ denote the number of respondents choosing the $r$ th option, and let $r_{\text {med }}$ denote the median value of $r$ from all respondents. The observed disagreement is then calculated as

$$
d_{\mathrm{obs}}=\sum_{r=1}^{r_{\max }} n_{r}\left|r_{\mathrm{med}}-r\right|,
$$

where $\left|r_{\text {med }}-r\right|$ is the weight for the $r$ th choice. The expected disagreement is calculated as

$$
d_{\exp }=\frac{n}{k} \sum_{r=1}^{r_{\max }}\left|\frac{k+1}{2}-r\right| .
$$

The coefficient of agreement $A$ is then calculated as the complement of the ratio of observed to expected disagreement:

$$
A=1-\frac{d_{\mathrm{obs}}}{d_{\exp }} .
$$




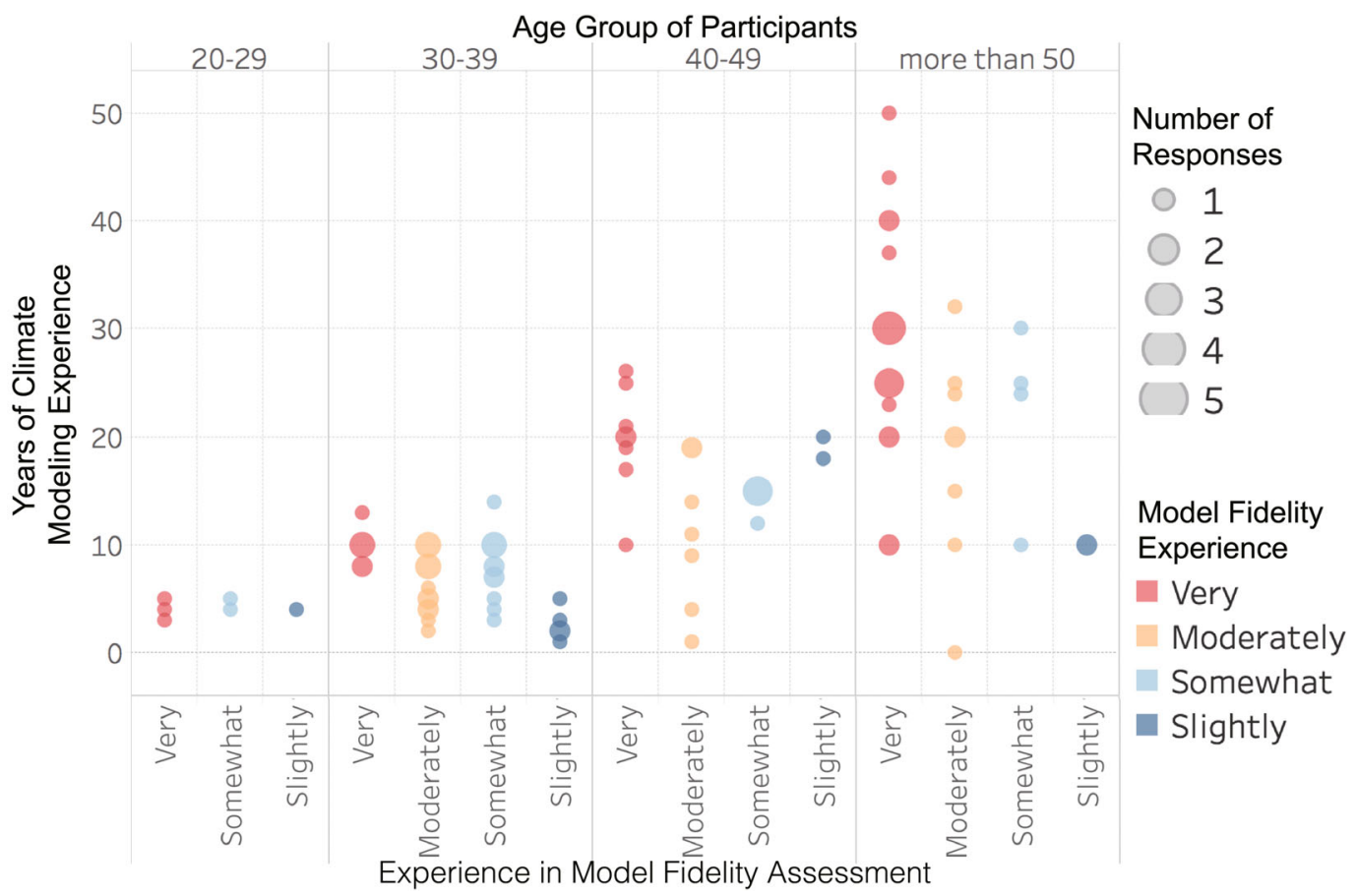

Fig. 1. Characteristics of survey participants.

For randomly distributed responses, $d_{\text {obs }}$ would be close to $d_{\text {exp }}$, and $A$ would be close to zero; while for perfect agreement, $d_{\mathrm{obs}}=0$ and $A=1$.

Because the value of $A$ is sensitive to the total number of respondents $N$, the value of $A$ is not comparable for subgroups of participants with different sizes. We performed additional significance testing to determine whether the degree of consensus was the same, or different, between our "high experience" and "low experience" groups, and/or between two survey drivers.

We test for statistically significant differences between two values of the coefficient of agreement for two groups of responses, $A_{1}$ and $A_{2}$, by performing a randomization test with the null hypothesis $H_{0}: A_{1}=A_{2}$. To perform this test, we take $l=1: 100$ random draws, without replacement, from the two groups of survey responses. For each $l$ th draw, we calculate the difference in the coefficient of agreement for the two groups, $d_{l}=\left|A_{1 l}-A_{2 l}\right|$. We then calculate the $p$-value for rejection of the null hypothesis, i.e., the probability that a difference in agreement larger than the observed mean could occur by chance:

$$
p=\frac{1}{100} \sum_{l=1}^{100} \begin{cases}1, & d_{l}>d_{l, \text { mean }}, \\ 0, & d_{l} \leqslant d_{l, \text { mean }}\end{cases}
$$

where $d_{l, \text { mean }}$ is the mean of all $d_{l}$.

\section{Survey results and discussion}

Here we report on selected analyses and results from the survey. We focus primarily on: (1) the degree of consensus among experts on the importance of different model variables; (2) how responsive experts' assessments of variable importance are to the defined scientific objectives; and (3) differences in expert ratings of variable importance between respondents with more climate modeling experience and those with less experience.

We also performed similar analyses comparing survey responses from model users and model developers. The responses of these two groups were statistically nearly identical, and so we do not report them in further detail.

\subsection{Importance of different variables to climate model fi- delity assessments across six Science Drivers}

In this section, we discuss expert ratings of variable importance for the six science drivers. In order to understand whether participants' responses differed depending on their degree of expertise, we first divided the participants into two experience groups: those who rated themselves as "very experienced" in evaluating model fidelity were placed into the "high experience" group $(N=36)$; all other participants were placed into the "low experience" group $(N=60)$.

We emphasize that our "low experience" group consists largely of working climate scientists over the age of 30 (95\%), with a median of 10 years of experience in climate modeling. In other words, our "low experience" group mostly consists not of laypersons, students or trainees, but of earlyto-mid-career climate scientists with moderate levels of experience in evaluating and tuning climate models. Our "high experience" group consists largely of mid-to-late career scientists: the majority are over the age of $50(53 \%)$, with a median of 20.5 years of experience in climate modeling. Researchers on the development of expertise have argued that roughly 10 years of experience are needed for the develop- 
ment and maturation of expertise (Ericsson, 1996); 86\% of our "high experience" group members have 10 years or more of climate modeling experience.

\subsubsection{Science Driver 1: How well does the model repro-} duce the overall features of the Earth's climate?

Our first Science Driver asked respondents to assess the importance of different variables to "the overall features of Earth's climate". We believe that this statement summarizes the primary aim of most experts when calibrating a climate model. However, experts' typical practices are likely to be influenced by factors such as the tools and practices used by their mentors and immediate colleagues, their disciplinary background, and their research interests. Such factors could contribute to differences in judgments of what constitutes a "good" model simulation. The aim of this Science Driver is to understand what experts prioritize when the goal is relatively imprecisely defined as optimizing the "overall features" of climate; these responses can then be contrasted with the more specific questions in the following five Science Drivers.

Figures 2 and 3 show the distribution of responses for each variable in Science Driver 1 for the high and low experience groups. Figure 4 (top) summarizes the mean and standard deviation of importance ratings for all variables in Science Driver 1. Overall, the variables most likely to be identified as "extremely important" were (in ranked order): rain flux $(N=31)$, 2-m air temperature $(N=28)$, longwave cloud forcing $(N=22)$, shortwave cloud forcing $(N=21)$, and sea level pressure $(N=20)$. The complete distributions of responses for all science drivers by experience group, together with statistical summary variables and significance tests, are shown in Tables S1-13.

The distribution and degree of consensus is similar between the two groups, with no statistically significant differences for any variable (see Supplementary Tables S4-S6). This suggests that once an initial level of experience is acquired, additional experience may not lead to significant differences in judgments about model fidelity.

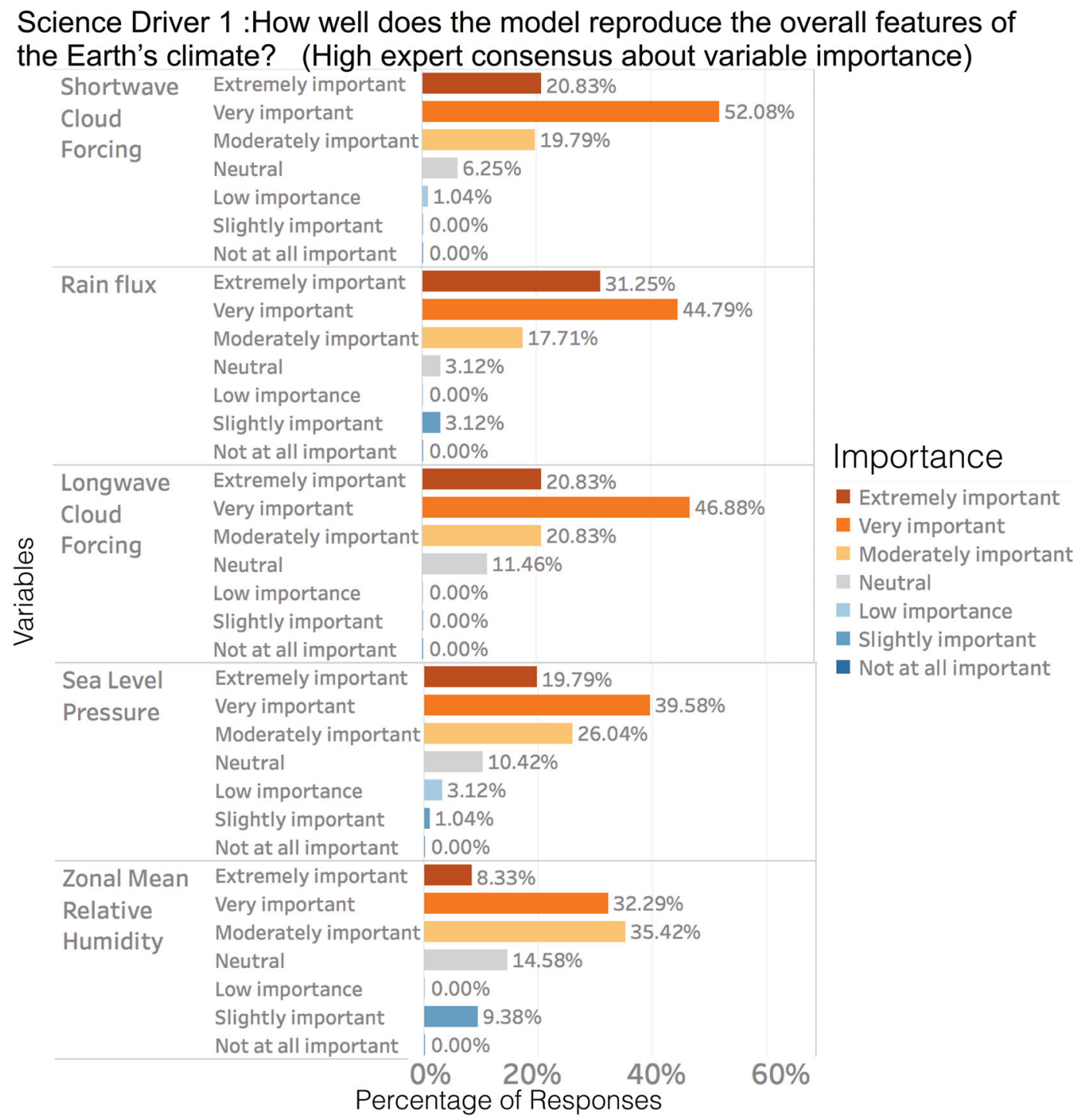

Fig. 2. Science Driver 1: distributions of importance ratings, ranked by consensus, as quantified by the coefficient of agreement $A$, for variables with high expert consensus about their importance. 


\section{Science Driver 1 :How well does the model reproduce the overall features of the Earth's climate? (Low expert consensus about variable importance)}

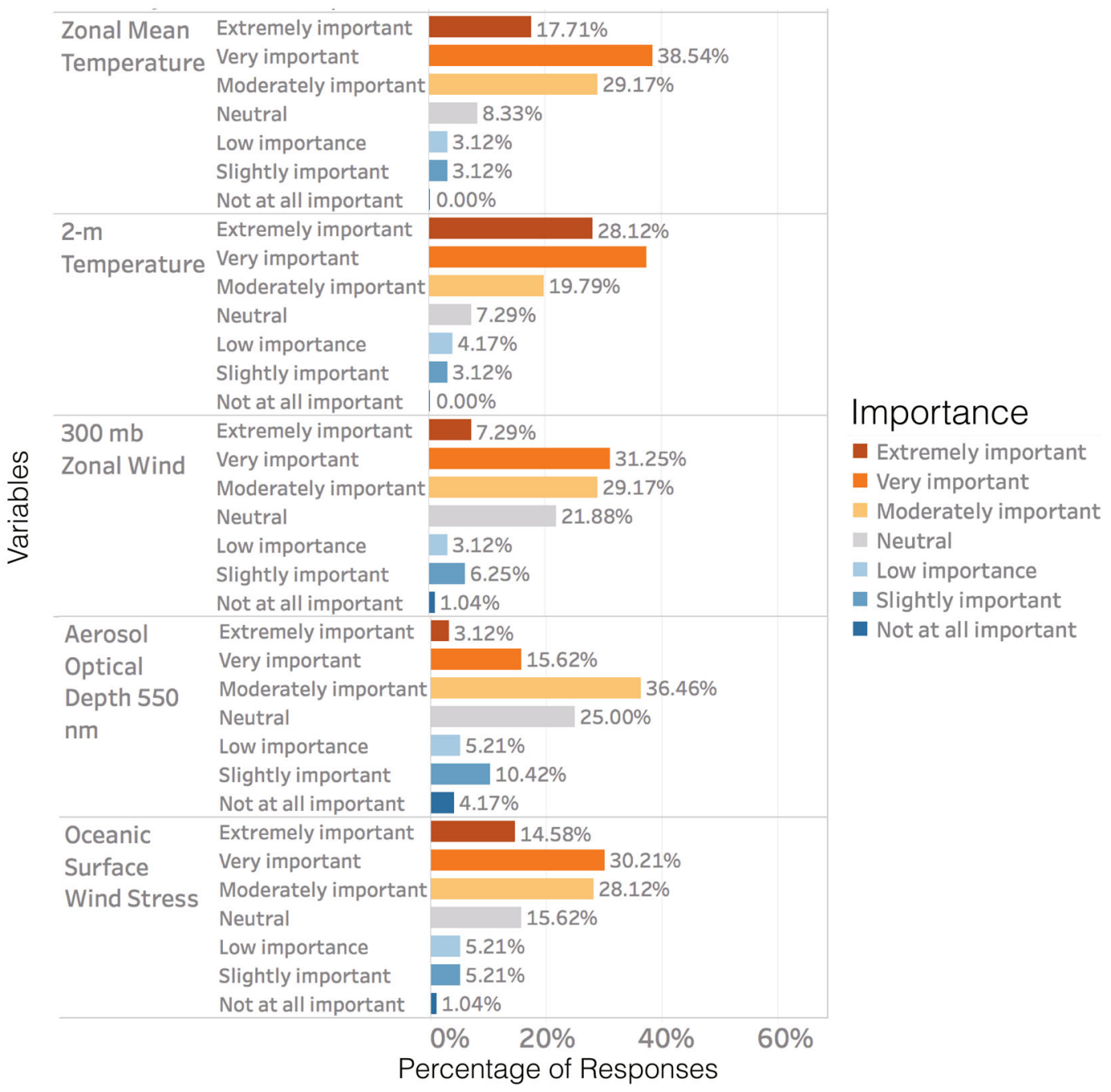

Fig. 3. As in Fig. 2 but for variables with low expert consensus about their importance.

It is instructive to examine which variables are the exceptions to this general rule; these exceptions hint at insights into where and how greater experience matters most in informing the judgments experts make about model fidelity. The distribution of responses of the high experience and low experience group differed for only one item in Science Driver 1 -the oceanic surface wind stress $(p<0.01)$; for this variable, the median response of the high and low experience groups was "very important" and "moderately important," respectively. We speculate that the high-experience group may be more sensitive to this variable due to (1) its critical importance to ocean-atmosphere coupling, and (2) awareness of the relatively high-quality observational constraints available from wind scatterometer data.

We also investigated the degree of consensus on the importance of different variables. We observe a clearly higher degree of consensus for some variables, compared to others. Across all participants (high and low experience groups together), there is a comparatively high degree of consensus on the importance of shortwave cloud forcing $(A=0.67)$, longwave cloud forcing $(A=0.62)$, and rain flux $(A=0.62)$. In particular, there is comparatively little agreement on the importance of oceanic surface wind stress $(A=0.39)$, due to the discrepancy between experience groups on this item, and on the aerosol optical depth (AOD; $A=0.42)$. The data we collected do not allow us to be certain of the reasoning behind importance ratings, but the lack of consensus on AOD importance is perhaps unsurprising in light of the high uncertainty associated with the magnitude of aerosol impacts on climate (Stocker et al., 2013), and recent controversies among climate modelers on the importance of aerosols to climate, or lack thereof (Booth et al., 2012; Stevens, 2013; Seinfeld et al., 2016).

\subsubsection{Science Driver 2: How well does the model repro- duce features of the global water cycle?}

Our second Science Driver included a comparatively limited number of variables related to the global water cycle 
Science Driver 1:

How well does the model reproduce the overall features of the Earth's climate?

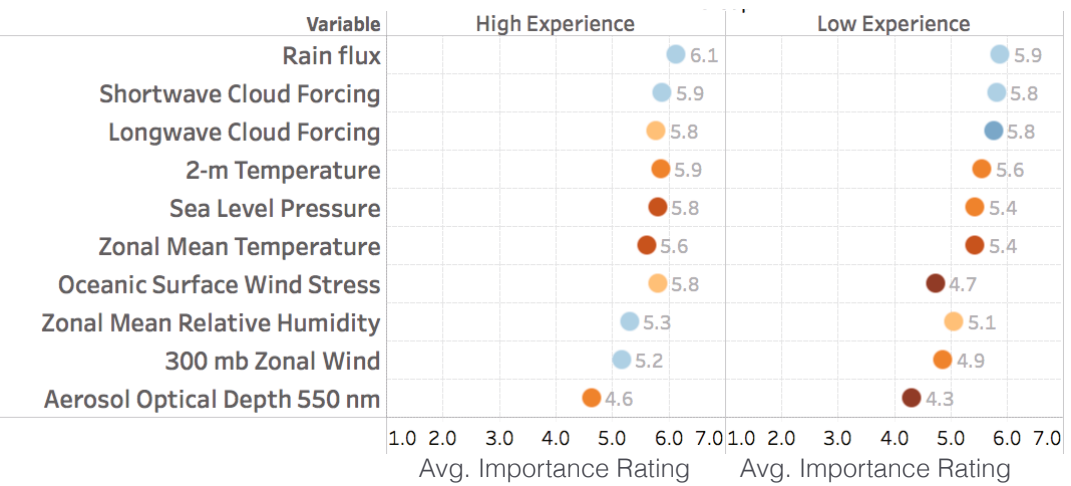

\section{Avg. Standard \\ Deviation}

Science Driver 2

How well does the model reproduce features of the global water cycle?

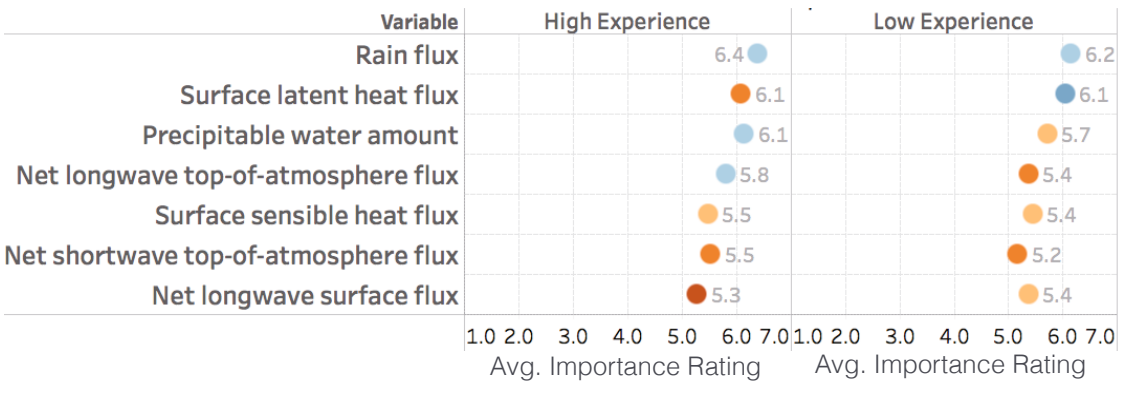

Science Driver 3 :

How well does the model simulate Southern ocean climate?

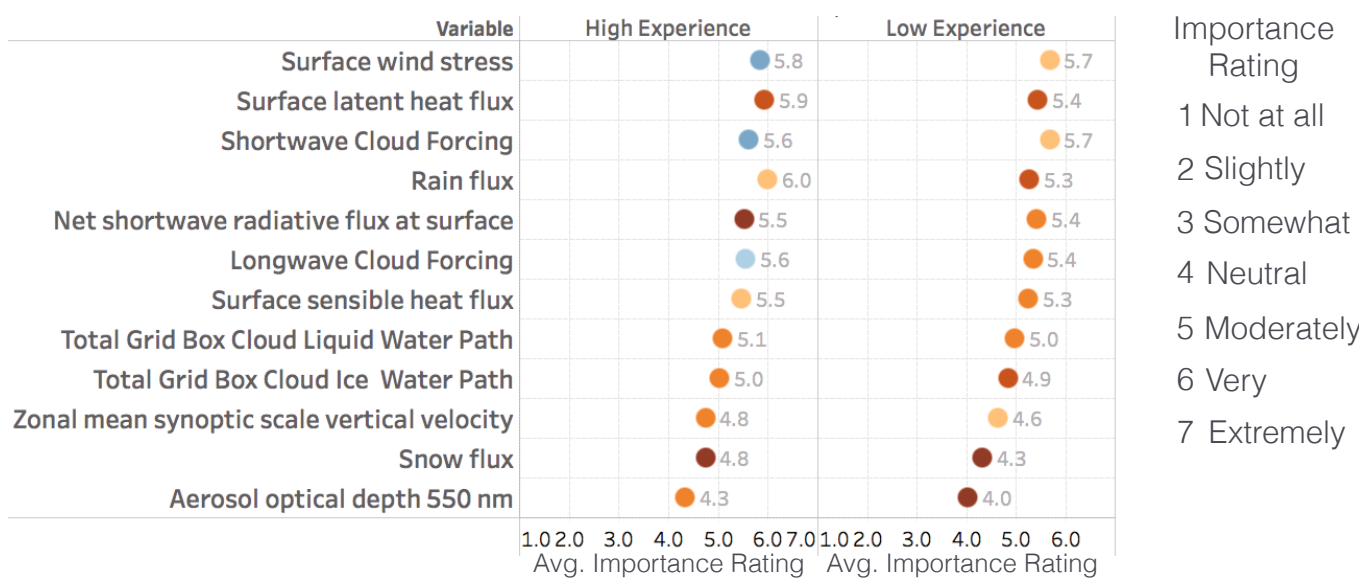

Fig. 4. Science Drivers 1-3: mean responses, high and low experience groups, ranked by overall mean response from all participants; color of dots indicates standard deviation of responses.

(Fig. 4: middle). These should be considered in combination with Science Driver 6, which addresses the assessment of simulated clouds using a satellite simulator (Fig. 5).

While the differences did not pass our criteria for statistical significance, we note a slight tendency for the high experience group to assign higher mean importance ratings to net TOA radiative fluxes and precipitable water amount. We speculate that this might be due to a slightly greater awareness of, and sensitivity to, observational uncertainties among the high experience group, expressed as a higher importance rating for variables with stronger observational constraints from satellite measurements. This interpretation is supported by the comment of one study participant (with 20 years' experience in climate modeling), who observed that "surface $\mathrm{LH}$ [latent heating] and SH [sensible heating] are not well constrained from obs[ervations]. While important, that means they aren't much use for tuning."

\subsubsection{Science Driver 3: How well does the model simulate Southern Ocean climate?}

For Southern Ocean climate, surface interactions that affect ocean-atmosphere coupling, including wind stress, latent heat flux (evaporation) and rain flux, together with shortwave cloud forcing, were identified as among the most important 
Science Driver 4:

How well does the model simulate important features of the water cycle in the Amazon watershed?

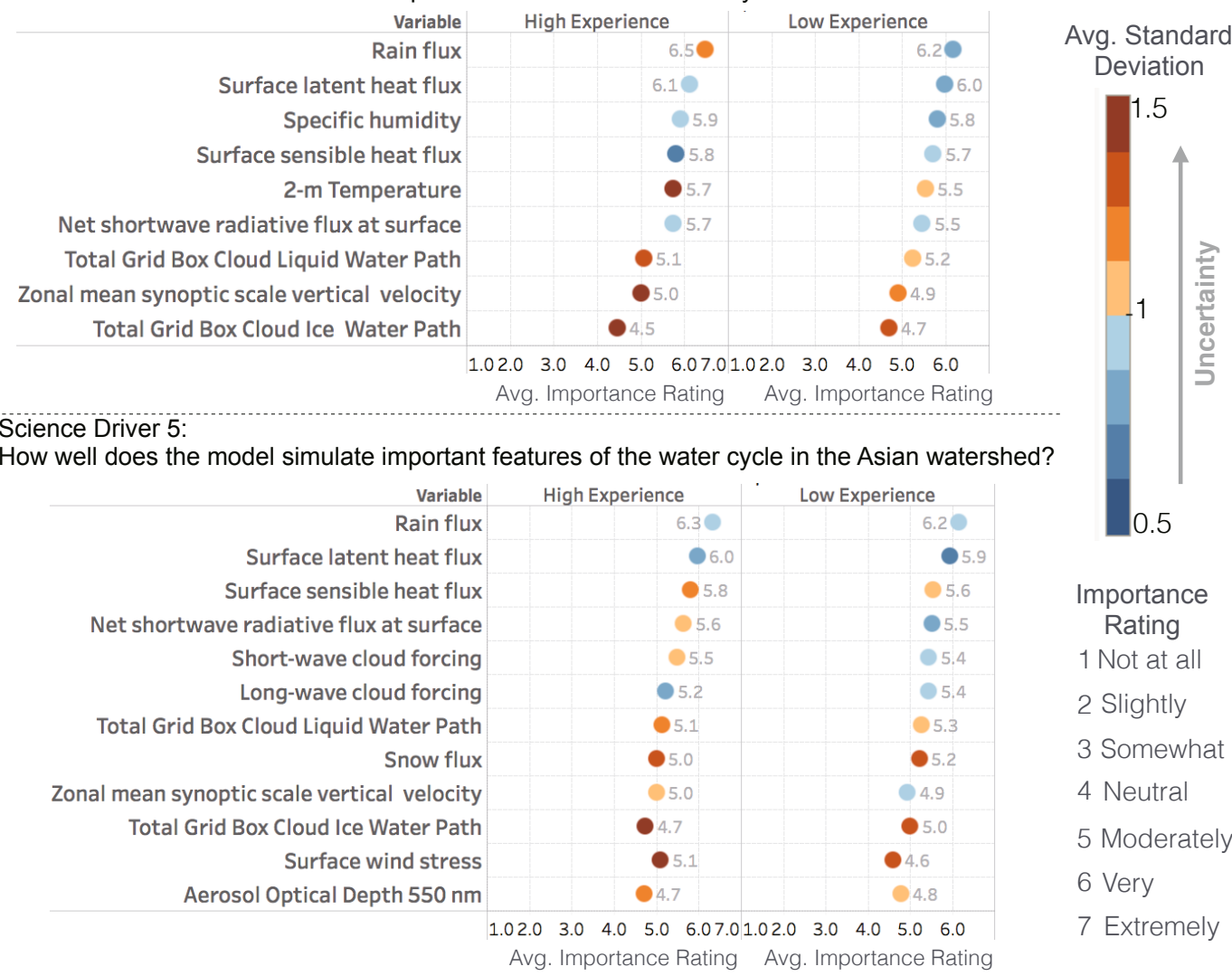

Fig. 5. Science Drivers 4-5: mean responses, high and low experience groups, ranked by overall mean response from all participants; color of dots indicates standard deviation of responses.

variables by our participants (Fig. 4: bottom).

The high experience group rated rain fluxes as more important (median: "very" important) compared to the low experience group (median: "moderately" important; probability of difference: $p=0.02$ ).

It is interesting to compare the responses with Science Driver 1, which included many of the same variables. For instance, for AOD, the low experience group assigned a lower mean importance for overall climate (mean: $4.32 ; \sigma: 1.41$ ) than for Southern Ocean climate (mean: 4.04; $\sigma: 1.49$ ); the high experience group assigned a higher mean importance for overall climate (mean: $4.64 ; \sigma: 1.16)$ than for Southern Ocean climate (mean: $4.34 ; \sigma: 1.13$ ).

The reasons for this discrepancy are unclear. One possibility is that the high experience group may be more aware that over the Southern Ocean, AOD provides a poor constraint on cloud condensation nuclei (Stier, 2016), and is affected by substantial observational uncertainties, with estimates varying widely between different satellite products.

3.1.4. Science Driver 4: How well does the model simulate important features of the water cycle in the Amazon watershed?

On Science Driver 4, which addresses the water cycle in the Amazon watershed (Fig. 5: top), participants identified surface sensible and latent heat flux, specific humidity, and rain flux as the most important variables for evaluation. It is possible that the more experienced group is more sensitive to the critical role of land-atmosphere coupling in the Amazonian water cycle. This interpretation would be consistent with the additional variables suggested by our survey participants for this science driver, which also focused on variables critical to land-atmosphere coupling, e.g. "soil moisture", "water recycling ratio", and "plant transpiration" (Supplementary Table S2). While the variables selected for the survey focused largely on mean thermodynamic variables, commenters also mentioned critical features of local dynamics in the Amazon region, such as surface topography and "wind flow over the Andes", "convection", and vertical velocity at $850 \mathrm{hPa}$.

\subsubsection{Science Driver 5: How well does the model simulate important features of the water cycle in the Asian wa- tershed?}

For Science Driver 5, focused on the Asian watershed, participants rated rain flux, surface latent heat flux, and net shortwave radiative flux at the surface as the most important variables (Fig. 5: bottom). For variables included in both Science Drivers, the order of variable importance was the same as in the Amazon watershed, but different than in the Southern Ocean; some of these differences will be discussed in section 3.3. Written responses again mentioned soil moisture $(3 \times)$ and moisture advection $(2 \times)$ as important variables 
missing from the list.

\subsubsection{Science Driver 6: How well does the model simulate the climate impact of clouds globally?}

The final Science Driver addressed the evaluation of cloud properties in the model (Fig. 6) using a satellite simulator, which produces simulated satellite observations and retrievals based on radiative transfer calculations in the model. "Very important" (6) was the most common response for all variables in Science Driver 6 (Supplementary Table S15).

While differences in responses between the two experience groups did not pass our bar for statistical significance, the high experience group selected "extremely important" more frequently than the low experience group for the "high level cloud cover" and "low cloud cover" items, which also had the highest mean importance ratings in this Science Driver.

Five participants indicated that longwave cloud forcing and shortwave cloud forcing should have been included, and one respondent noted "A complete vertical distribution of cloud properties would be even more interesting than "low", "medium" and "high" cloud cover. Cloud particle size and number would also be interesting." Another responded that "cloud fraction is a model convenience but is quite arbitrary."

\subsection{Impact of experience on judgments of variable impor- tance}

We hypothesized that: (H1) respondents with less experience in climate modeling would differ from more experienced respondents in their judgments of relative variable importance; and (H2) Respondents with greater experience in climate modeling would exhibit greater consensus in their judgments of the importance of different variables.

(H1): Using a Chi-squared significance test (details in the Supplementary Material), we find support for differences in assessment of variable importance by high and low experience groups, but only for certain selected variables. Compared to the low experience group, the high experience group rated ocean surface wind stress as more important to evaluation of global climate (Science Driver 1) and rain flux as more important to evaluation of Southern Ocean climate (Science Driver 3).

Some other differences are observable between the two groups (see Supplementary Tables S10-S15), but did not meet our criteria for significance; it is possible that additional differences would emerge if a larger survey population could be attained.

$(\mathrm{H} 2)$ : We find no statistically significant differences in degree of consensus between the high and low experience groups.

The lack of large differences in responses between the high and low experience groups suggests that variations in importance ratings are mainly driven by factors that are unrelated to the amount of experience the scientists have. Examples could include the specific subdiscipline of the individual expert, or the practices and research foci that are common in their particular research community or geographic area. This result also suggests that expertise in climate model evaluation may reach a plateau after a certain level of proficiency is attained, with additional experience leading to only incremental changes in expert evaluations and judgments. One possible reason for this is that the process of model evaluation is

\section{Science Driver 6:} How well does the model simulate the climate impact of clouds globally?

\begin{tabular}{|c|c|c|}
\hline Variable & High Experience & Low Experience \\
\hline Low Cloud Cover & 6.30 & $6.1 \bullet$ \\
\hline High-Level Cloud Cover & 6.10 & 5.8 \\
\hline Total cloud fraction & 6.10 & - 5.8 \\
\hline Low-level cloud cover over oceans from optically .. & 5.8 & 5.8 \\
\hline Thick cloud fraction (tau >9.4) & - 5.8 & 5.8 \\
\hline Middle-Level Cloud Cover & - 5.6 & 5.5 \\
\hline Thin cloud fraction $(9.4>$ tau $>1.3)$ & 5.5 & 5.5 \\
\hline Total Grid Box Cloud Liquid Water Path over ocean & 5.5 & 5.5 \\
\hline & .06 .0 & 5.06 .0 \\
\hline
\end{tabular}

Avg. Importance Rating Avg. Importance Rating

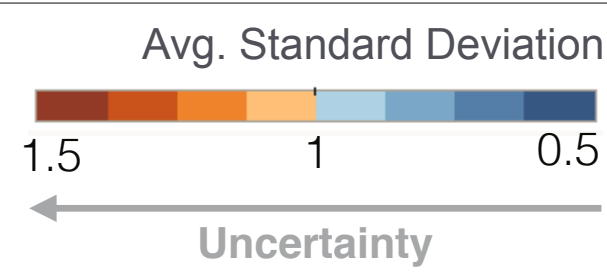

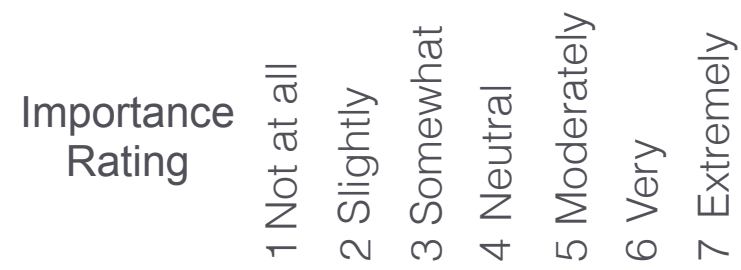

Fig. 6. Science Driver 6: mean responses, high and low experience groups, ranked by overall mean response from all participants; color of dots indicates standard deviation of responses. 
constantly evolving as updated model versions incorporate additional processes and improvements, new observational datasets become available, and new tools are developed to support the evaluation process. As a result, climate scientists continually need to update their understanding about climate models and their evaluation to reflect the current state-of-theart. Another possible explanation is that the culture of the climate modeling community may promote an efficient transfer of knowledge, as more experienced scientists offer training and advice to less experienced colleagues and to other research groups, shortening the learning curve of new scientists entering the field.

\subsection{Impact of Science Drivers on judgments of variable importance}

We expected that survey participants would rate the importance of the same model variables differently depending on the science goals, and indeed this is what we found. In this section, we focus on the ratings from the high experience group, but results from the low experience group are similar.

For instance, rain flux was rated as less important to evaluation of the Southern Ocean (mean: $6.00 ; \sigma: 1.12$ ) than to global climate (mean: $6.14 ; \sigma: 0.92$ ) or the Asian watershed (mean: 6.32; $\sigma: 1.00$ ), while shortwave and longwave cloud forcing were rated as less important to the Asian watershed (shortwave: mean: 5.48; $\sigma$ : 0.84; longwave: mean: 5.23; $\sigma: 1.01)$ than to global climate (shortwave: mean: $5.89 ; \sigma$ : 1.02; longwave: mean: $5.78 ; \sigma: 1.02$ ) or Southern Ocean climate (shortwave: mean: $5.63 ; \sigma: 0.86$; longwave: mean: 5.56; $\sigma: 0.90)$. Surface wind stress was rated more important in the Southern Ocean (mean: 5.84; $\sigma: 1.30$ ), and less important in the Asian watershed (mean: $5.10 ; \sigma: 1.33$ ), compared to its importance to global climate evaluation (mean: $5.81 ; \sigma: 1.02)$. While total cloud liquid water path was rated as equally important in the Southern Ocean (mean: 5.09; $\sigma$ : 1.10), Amazon watershed (mean: 5.06; $\sigma: 1.29$ ), and Asian watershed (mean: $5.13 ; \sigma: 1.13$ ), total cloud ice water path was rated as less important to the evaluation of the model in the Amazon watershed (mean: 4.45; $\sigma: 1.52$ ) and Asian watershed (mean: $4.74 ; \sigma: 1.22$ ), compared to the Southern
Ocean (mean: $5.03 ; \sigma: 1.13$ ).

These differences indicate that experts adjust the importance assigned to different metrics depending on the science question or region they are focusing on. As a result, we recommend that future work focused on understanding or quantifying expert judgments of model fidelity should always be explicit about the scientific goals for which the model under assessment will be evaluated.

\subsection{Perceived barriers to systematic quantification of model fidelity}

We also explored the community's perceptions about the current obstacles to systematic quantification of model fidelity (Fig. 7). Survey participants identified the lack of robust statistical metrics (28\%) and lack of analysis tools (10\%) as major barriers, with $17 \%$ selecting "all of the above".

Many participants selected the option "Other" and contributed written comments. We grouped these into qualitative categories of responses. The most commonly identified issues related to:

- Lacking or inadequate observational constraints and error estimates for observations $(8 \times)$;

- Laboriousness of the tuning process $(7 \times)$; and

- Challenges associated with identifying an appropriate single metric of model fidelity $(7 \times)$.

On the final point, many of the comments focused on the risk of oversimplifying the analysis and evaluation of models: "Focusing on single metrics over simplifies the analysis too much to be useful. It is often hard to identify good vs. bad because one aspect works while others don't, and different models have different trade offs." "No one metric tells the whole story; this may lead to false confidence in model fidelity." Another commenter noted that "it's very hard to create a single metric that accurately encapsulates subjective judgments of many scientists." Finally, several respondents noted other barriers, including a perceived lack of sufficient expertise in the community, a perception that some widespread practices are inadequate or inappropriate for model evaluation, and a lack of sufficient attention to model sensitivities, as opposed to calibration with respect to present-day mean climate.

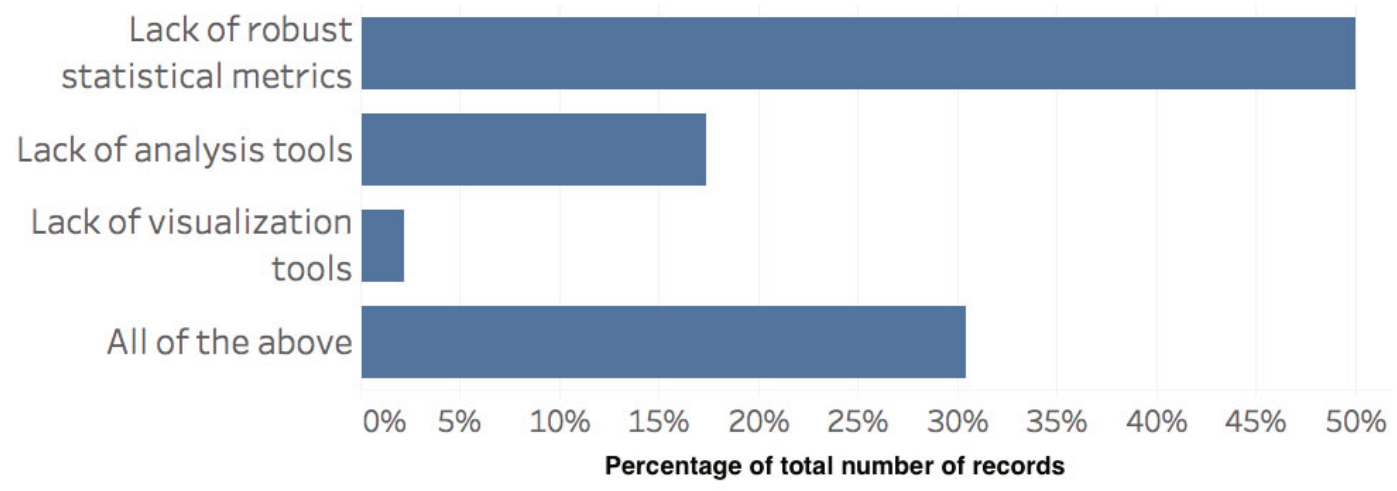

Fig. 7. Perceived barriers to systematic quantification of model fidelity. Answers were selected from a predetermined list in response to the prompt: "Which one among the following, do you feel, is the biggest barrier towards systematic quantification of model fidelity?" 


\section{Prospects for synthesizing expert assess- ments and objective model fidelity metrics}

As discussed in section 1, there are many potential applications for a climate model index that summarizes the model's fidelity with respect to a particular science goal. However, one challenge is that an assessment of which models most resemble the observations depends in part on which observed variables are evaluated, and how much relative importance is assigned to each of them. A model fidelity index can be conceptualized as a weighted average of different objective metrics (Eq. 1), but different experts might reasonably make different choices in assigning values to the weights, resulting in models potentially being ranked differently by different experts, as illustrated in Fig. 8. Furthermore, the information that experts implicitly use and the relative importance they assign to different aspects of the model's fidelity when evaluating actual model output, likely differs from their explicit statements about evaluation criteria. A systematic approach is needed to understand which information experts actually use in evaluating models, how much consensus exists among experts about variable importance when evaluating real model output, and how sensitive a proposed model fidelity index would be to differences in these judgments between experts.

The survey described in this paper represents a first step towards building that understanding. It also provides baseline information that will inform and be used in analysis of a

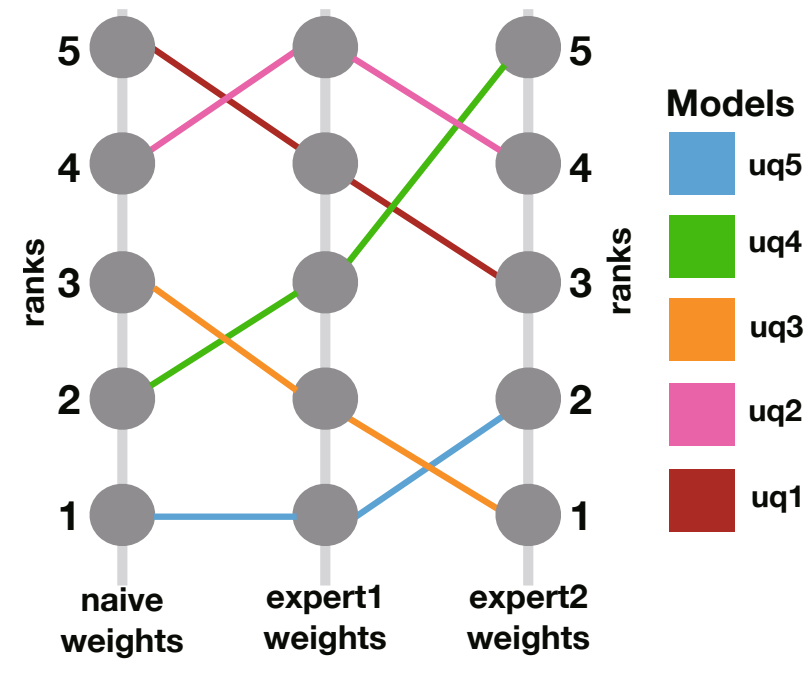

Fig. 8. Illustration of the concept of overall model fidelity rankings and their sensitivity to expert weights. Consider the pair of models uq1 and uq2, where the overall fidelity of the model is evaluated as a weighted mean of several component scores. If uq1 performs better than uq2 on some component scores, but worse on others, the ranking of these models according to their overall mean fidelity metric will be sensitive to how strongly each component metric is weighted. In this example, the rankings of several models using "naive weights" (unweighted average) are compared to rankings that use importance weights derived from the responses of two different experts in our survey. second planned study, in which experts will be invited to evaluate the output from real model simulations. This mock model assessment exercise will enable us to address additional questions, such as: (1) How much consensus exists among experts when evaluating the fidelity of actual model simulations (as opposed to assessing variable importance in the abstract)? (2) can an index $I_{\text {inferred }}$ be constructed by using experts' assessments of real model output to infer the

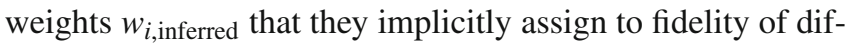
ferent model variables? (3) Do the weights $w_{i \text {,inferred }}$ that are inferred from experts' assessments of real model output agree or disagree with the relative importance that experts assigned to different variables a priori, as reported in this study?

\section{Summary and conclusions}

In this article we report results from a large community survey on the relative importance of different variables in evaluating a climate model's fidelity with respect to a particular science goal. We plan to use the results of this study to inform the development of a follow-up study in which experts are invited to evaluate actual model outputs.

We show that experts' rankings are sensitive to the scientific objectives. For instance, surface wind stress was rated as among the most important variables in evaluation of Southern Ocean climate, and among the least important in evaluation of the Asian watershed. This suggests the possibility and utility of designing different and unique collections of metrics, tailored to specific science questions and objectives, while accounting explicitly for uncertainty in variable importance.

We find no statistically significant differences between rankings provided by model developers and model users, suggesting some consistency between the developer and user communities' understanding of appropriate evaluation criteria. We also find that our "high experience" group, consisting mostly of senior scientists with many years of climate modeling experience, and our "low experience" group, consisting mostly of early and mid-career scientists, were in agreement about the importance of most variables for model evaluation. However, within each group, there are also substantial disagreements and diversity in responses. The level of consensus is particularly low for AOD, which some participants rated as "extremely important" and others rated as "not at all important." Additionally, in our survey sample, greater experience with evaluating model fidelity was not associated with greater consensus about the importance of different variables in model evaluation, and led to only minor changes in estimates of variable importance, i.e., to small changes in the frequency distribution of importance ratings, which are only statistically significant for a small number of variables.

It is important to note that when experts' responses on this survey differ, it does not necessarily imply that their evaluations of actual climate models would also differ. We anticipate that experts perform actual model evaluations in a more holistic manner and draw on much broader information than was included in this survey. In order to make initial progress 
on this extremely complex topic, we limited the scope of the study to evaluation of global mean climate, but the timedependent behavior of the system is also critical to assess, as well as features of the coupled climate system. Future research should extend this approach to include evaluation of diurnal and seasonal cycles; multi-year modes of climate variability such as ENSO, QBO, and PDO; extreme weather events; frequency of extreme precipitation; and other timedependent features of the climate system. Other, more complex metrics of model fidelity could also be considered, e.g., object-based verification approaches, and scale-aware metrics that would be robust to changes in model resolution.

Several study participants noted that issues related to observational datasets continue to be a major challenge for model evaluation. This includes logistical issues, such as their availability through a centralized repository, in standardized formats, and in updated versions as new data become available. However, more fundamentally, the limitations of observational constraints continue to be a major obstacle, including the lack of observations of certain key model variables, and the lack of estimates of the observational uncertainty for many datasets. Climate model evaluation efforts could also benefit from the increased adoption of metrics and diagnostic visualizations that directly incorporate information on observational uncertainty and natural variability, providing greater transparency and richer contextual information to users of these tools.

The labor-intensiveness of model evaluation efforts was noted by several survey participants, and is well-known to most scientists familiar with climate model development. Climate modeling centers invest an enormous amount of computational and human resources into model tuning. At a rough estimate, tuning a coupled climate model requires the efforts of about five full-time equivalent (FTE) scientists and engineers for each major model component (atmosphere, ocean and sea-ice, and land) as well as five FTEs for the overall software engineering and tuning of the coupled system. An intense tuning effort for a new major version of a coupled climate model may last for about one year and be repeated every five years, for an average investment of four FTEs per year. Globally, there are at least 26 major climate modeling centers (the number that participated in CMIP5 project: http://cmip-pcmdi.llnl.gov/cmip5/availability.html), of which five are located in the United States (DOE-ACME, NASAGISS, NASA-GMAO, NCAR, NOAA-GFDL). Assuming that the typical cost to support a staff scientist at a climate modeling center is about $\$ 300$ thousand per year (including salary, fringe, and overhead expenses), we estimate that the amount of money spent annually on the human effort involved in climate model tuning is roughly $\$ 6$ million in the United States and \$31.2 million globally.

If appropriate quantitative metrics can be developed that meaningfully capture the criteria important in a comprehensive model assessment, then algorithms could be applied to partially automate the calibration process, for instance by identifying an initial subset of model configurations that produce plausible climates, subject to further manual inspection by teams of experts. Further work is needed to assess the feasibility of such an approach; but if successful, similar approaches could be valuable in the development not only of global climate models, but also of regional weather models, large eddy simulations, and other geophysical and complex computational models in which multiple aspects of fidelity must be assessed and weighed against each other.

We suggest that a closer integration of objectively computed metrics with expert understanding of their relative importance has the potential to dramatically improve the efficiency of the model calibration process. The concise variable lists and community ratings reported in this study provide a snapshot of current expert understanding of the relative importance of certain aspects of climate model behavior to their evaluation. This information will be informative to the broader climate research community, and can serve as a starting point for the development of more sophisticated evaluation and scoring criteria for global climate models, with respect to specific scientific objectives.

Acknowledgements. The authors would like to express their sincere gratitude to everyone who participated in the survey described in this paper. While privacy restrictions prevent us from publishing their identities, we greatly appreciate the time that many busy individuals have taken, voluntarily, to contribute to this research. We would like to thank Hui WAN, Ben KRAVITZ, Hansi SINGH, and Benjamin WAGMAN for helpful comments and discussions that helped to inform this work. This research was conducted under the Laboratory Directed Research and Development Program at PNNL, a multi-program national laboratory operated by Battelle for the U.S. Department of Energy under Contract DEAC05-76RL01830.

Open Access This article is distributed under the terms of the Creative Commons Attribution License which permits any use, distribution, and reproduction in any medium, provided the original author(s) and the source are credited.

Electronic supplementary material Supplementary material is available in the online version of this article at https://doi.org/ 10.1007/s00376-018-7300-x.

\section{REFERENCES}

Booth, B. B. B., N. J. Dunstone, P. R. Halloran, T. Andrews, and N. Bellouin, 2012: Aerosols implicated as a prime driver of twentieth-century North Atlantic climate variability. Nature, 484, 228-232, https://doi.org/10.1038/nature10946.

Braverman, A., N. Cressie, and J. Teixeira, 2011: A likelihoodbased comparison of temporal models for physical processes. Statistical Analysis and Data Mining: The ASA Data Science Journal, 4, 247-258, https://doi.org/10.1002/sam.10113.

Ericsson, K., 1996: The Road to Expert Performance: Empirical Evidence from the Arts and Sciences, Sports, and Games. Lawrence Erlbaum Associates, 369 pp.

Gleckler, P. J., K. E. Taylor, and C. Doutriaux, 2008: Performance metrics for climate models. J. Geophys. Res., 113, D06104, https://doi.org/10.1029/2007JD008972. 
Herger, N., G. Abramowitz, R. Knutti, O. Angélil, K. Lehmann, and B. M. Sanderson, 2017: Selecting a climate model subset to optimise key ensemble properties. Earth System Dynamics, 9, 135-151, https://doi.org/10.5194/esd-9-135-2018.

Hourdin, F., and Coauthors, 2017: The art and science of climate model tuning. Bull. Amer. Meteor. Soc., 98, 589-602, https://doi.org/10.1175/BAMS-D-15-00135.1.

Knutti, R., J. Sedláček, B. M. Sanderson, R. Lorenz, E. M. Fischer, and V. Eyring, 2017: A climate model projection weighting scheme accounting for performance and interdependence. Geophys. Res. Lett., 44, 1909-1918, https://doi.org/10.1002/ 2016GL072012.

Min, S. K., and A. Hense, 2006: A Bayesian approach to climate model evaluation and multi-model averaging with an application to global mean surface temperatures from IPCC AR4 coupled climate models. Geophys. Res. Lett., 33, L08708, https://doi.org/10.1029/2006GL025779.

Nosedal-Sanchez, A., C. S. Jackson, and G. Huerta, 2016: A new test statistic for climate models that includes field and spatial dependencies using Gaussian Markov random fields. Geoscientific Model Development, 9, 2407-2414, https://doi.org/ 10.5194/gmd-9-2407-2016.

Qian, Y., and Coauthors, 2015: Parametric sensitivity analysis of precipitation at global and local scales in the Community Atmosphere Model CAM5. Journal of Advances in Modeling Earth Systems, 7, 382-411, https://doi.org/10.1002/2014 MS000354.

Qian, Y., and Coauthors, 2016: Uncertainty quantification in climate modeling and projection. Bull. Amer. Meteor. Soc., 97, 821-824, http://dx.doi.org/10.1175/BAMS-D-15-00297.1.

Reichler, T., and J. Kim, 2008: How well do coupled models simulate today's climate? Bull. Amer. Meteor. Soc., 89, 303-311, https://doi.org/10.1175/BAMS-89-3-303.
Riffenburgh, R. H., and P. A. Johnstone, 2009: Measuring agreement about ranked decision choices for a single subject. The International Journal of Biostatistics, 5, https://doi.org/ $10.2202 / 1557-4679.1113$.

Seinfeld, J. H., and Coauthors, 2016: Improving our fundamental understanding of the role of aerosol-cloud interactions in the climate system. Proceedings of the National Academy of Sciences of the United States of America, 113, 5781-5790, https://doi.org/10.1073/pnas.151404311.

Stevens, B., 2013: Aerosols: Uncertain then, irrelevant now. $\mathrm{Na}$ ture, 503, 47-48, https://doi.org/10.1038/503047a.

Stier, P., 2016: Limitations of passive remote sensing to constrain global cloud condensation nuclei. Atmospheric Chemistry and Physics, 16, 6595-6607, https://doi.org/10.5194/acp-166595-2016.

Stocker, T. F., and Coauthors, 2013: Climate Change 2013: The Physical Science Basis. Contribution of Working group I to the Fifth Assessment Report of the Intergovernmental Panel on Climate Change. Cambridge University Press, 1535 pp, https://doi.org/10.1017/CBO9781107415324.

Suckling, E. B., and L. A. Smith, 2013: An evaluation of decadal probability forecasts from state-of-the-art climate models. $J$. Climate, 26, 9334-9347, https://doi.org/10.1175/JCLI-D-1200485.1.

Yang, B., and Coauthors, 2013: Uncertainty quantification and parameter tuning in the CAM5 Zhang-McFarlane convection scheme and impact of improved convection on the global circulation and climate. J. Geophys. Res., 118, 395-415, https://doi.org/10.1029/2012JD018213.

Zhang, T., L. Li, Y. Lin, W. Xue, F. Xie, H. Xu, and X. Huang, 2015: An automatic and effective parameter optimization method for model tuning. Geoscientific Model Development, 8, 3579-3591, https://doi.org/10.5194/gmd-8-3579-2015. 